\title{
A Review on Fall Detection Systems Using Data from Smartphone Sensors
}

\author{
Md. Milon Islam ${ }^{1 *}$, Nieb Hasan Neom ${ }^{1}$, Md. Samir Imtiaz ${ }^{1}$, Sheikh Nooruddin ${ }^{1}$, Md. Repon Islam ${ }^{1}$, Md. Rabiul Islam ${ }^{2}$ \\ ${ }^{1}$ Department of Computer Science and Engineering, Khulna University of Engineering \& Technology, Khulna-9203, Bangladesh \\ ${ }^{2}$ Department of Electrical \& Electronic Engineering, Bangladesh Army University of Engineering \& Technology, Natore-6431, \\ Bangladesh
}

Corresponding Author Email: milonislam@cse.kuet.ac.bd

https://doi.org/10.18280/isi.240602

Received: 15 July 2019

Accepted: 5 October 2019

\section{Keywords:}

fall, fall detection, smartphone, threshold based system, machine learning based system

\begin{abstract}
Accidental falls have become one of the most frequent general health issues in recent years due to the rate of occurrence. Individuals aged above 65 are more prone to accidental falls. Accidental falls result in severe injuries such as concussion, head trauma, physical disabilities even to deaths in serious cases if the patients are not rescued in time. Thus, researchers are focusing on developing fall detection systems that facilitate the detection and quick rescue of fall victims. The smartphone-based fall detection systems use various built-in sensors of smartphones mostly Tri-axial accelerometer, magnetometer, gyroscope, and camera. The majority of the systems employ threshold based algorithms (TBA). Some systems use machine learning (ML) based algorithms or a combination of ML and TBA based algorithms to detect falls. Each of these types of systems has its trade-offs. The goal of this paper is to review fall detection systems based on data from smartphone sensors that employ either one of TBA, ML or combination of both. We also present the taxonomy based on systematic comparisons of existing studies for smartphone-based fall detection solutions.
\end{abstract}

\section{INTRODUCTION}

According to the Cambridge dictionary, a fall is to suddenly go down onto the ground or towards the ground without intending to or by accident [1]. Sudden collapse or fall is defined as an unintended and abnormal change in the posture of the body going down to the ground.

Every year, a huge number of individuals especially those who are 65 or more experience falls. More than $25 \%$ of elderly falls every year [2], but only half or less consult the doctors [3]. Falling once doubles the odds of falling again [4]. 20\% of falls cause fatal injury, for example, head trauma, or complex bone fracture $[5,6]$. A fall is prone to happen mostly at home environments such as living environment is usually filled with possible fall hazards [7] such as poor brightness of light, clutter, obstructed paths, pets, wet or greasy floors, unstable home appliance, etc. [8]. Elderly who suffer from neurological diseases like epilepsy and dementia [9] is more likely to fall than the average elderly. Every year, the number of new dementia patients reach 7 million in Europe alone, and the rate is predicted to nearly double every two decades [10]. Every year, fall injuries force the doctors to treat nearly 3 million elderly in emergency sections of hospitals [11]. As indicated by the World Health Organization (WHO), Falling is the subsequent driving reason behind accidental or unintentional injury related deaths worldwide. Every year about 6, 46,000 people die from fall injuries worldwide, out of which more than $80 \%$ are from low- and middle-income countries. Each year nearly 37.3 million falls occur that are serious enough to require medical support [12].

Fall detection and prevention systems have been extensively researched which has resulted in numerous methods of detecting falls. But no specific method was standardized or accepted globally [13]. We can classify these into surveillance systems, smartphone-based systems, and wearable devices based systems. Surveillance systems are normally computer vision based. Some surveillance-based systems employ IR arrays, microphone array systems, doppler radars, etc. Three basic functional sub-systems are required for most fall detection systems. These are data-reading/ sensing sub-system, data-processing sub-system, and datacommunication sub-system. In the case of a smartphone, the accelerometer, gyroscope, and camera can serve as the datasensing sub-system, and microcontrollers, microprocessors, ram, etc. can be used as the data-processing sub-system, and Bluetooth, mobile internet, wireless fidelity (Wi-Fi) technologies can perform communication-related tasks. All these basic criteria can be fulfilled by the android operating system based smartphones. Moreover, the computational capacity of smartphones is increasing day by day and so it is vastly used by researchers in recent years. People don't like or forget to carry extra devices or wearable devices with them. Additionally, there is a social stigma related to using externally visible devices for health purposes. Smartphones are a feasible solution to this problem because smartphones are less obtrusive items [14]. The number of mobile phone users in the world is predicted to pass the five billion mark by 2019 [15]. The number of smartphone users worldwide surpassed 3 billion [16]. By now, almost every adult person has a smartphone and the elderly are no different. Among the builtin sensors, the accelerometer is the single most used sensor for developing fall detection systems and gyroscope comes subsequently in the list. Accelerometers measure the acceleration that occurs during the fall and gyroscope is used to measure the angular displacement. The available smartphone based public motion datasets [17] mostly used 
smartphone sensors to record fall and ADL motions. Commonly used smartphone accelerometers have a range of \pm $2 \mathrm{~g}$ whereas industrial micro-electromechanical systems (MEMS) accelerometers have a range of up to $\pm 40 \mathrm{~g}$ [17]. Mellone et al. [18] and Albert et al. [19] compared the performance of built-in smartphone accelerometers and other dedicated or external sensing devices. It was found that the smartphone accelerometers were versatile enough for detecting fall and ADL motions. Vogt et al. [20] used smartphone accelerometers to accurately characterize free fall time. Thus, it can be stated that smartphone sensors have enough accuracy and sensitivity to properly classify human motion. Smartphone sensor based fall detection systems mostly use two types of algorithms. Most of the methods use threshold based algorithms (TBA). However, with the advent of sophisticated machine learning algorithms and the increased processing capabilities of smartphones, the use of machine learning algorithms to develop smartphone based fall detection systems is increasing rapidly. Although TBA based techniques require less computation power in comparison to ML based techniques, the latter provides better accuracy. The power consumption of various fall detection systems has been extensively discussed in previous researches [21-25]. To overcome the limitations of a single technique, a combination of TBA and ML based systems are being widely developed.

Figure 1 demonstrates a general block diagram of a fall detection system. The smartphone solely does the job of sensing, processing and communication device. As soon as a fall is spotted by the system, the corresponding message is been sent to authorized persons.

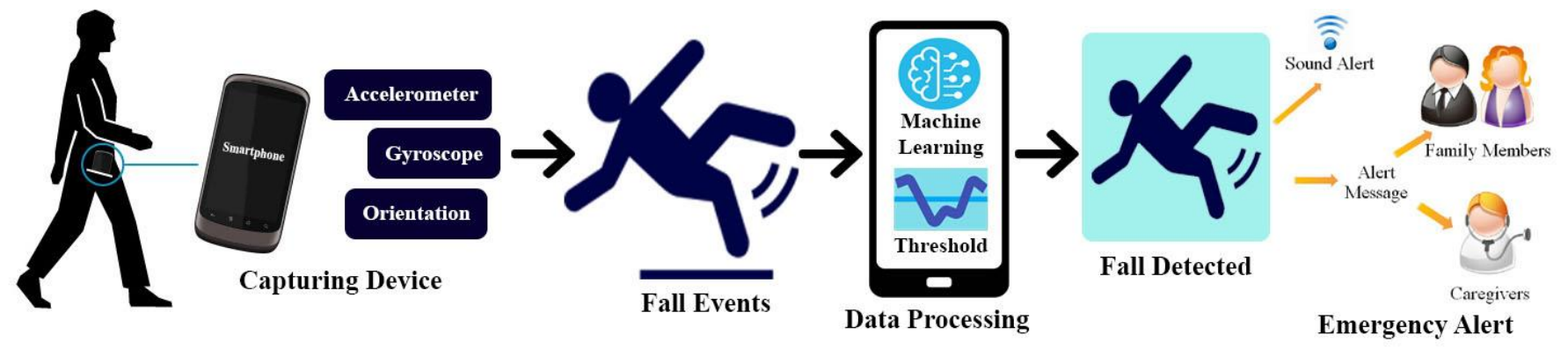

Figure 1. Fall detection system visualization

In this paper, we reviewed the fall detection systems based on the type of algorithm used. The systems were reviewed considering the following issues: the architecture of the systems, the used techniques, the performance, and the merits and demerits. We also proposed a taxonomy that categorizes the reviewed fall detection systems.

The remainder of this paper is presented as follows. Section 2 discusses the literature on fall detection systems respectively including the taxonomy and performance analysis for the systems. Subsection 2.1 reviews TBA-based fall detection systems. Subsection 2.2 demonstrates ML-based fall detection systems. The TBA-ML combined fall detection systems are reviewed in subsection 2.3. Section 3 presents a detailed discussion and summary. Finally, Section 4 concludes this review.

\section{LITERATURE REVIEW ON FALL DETECTION}

We can classify fall detection systems into three major types based on the algorithms used to distinguish between a fall and no-fall. Figure 2 simply demonstrates the classification.

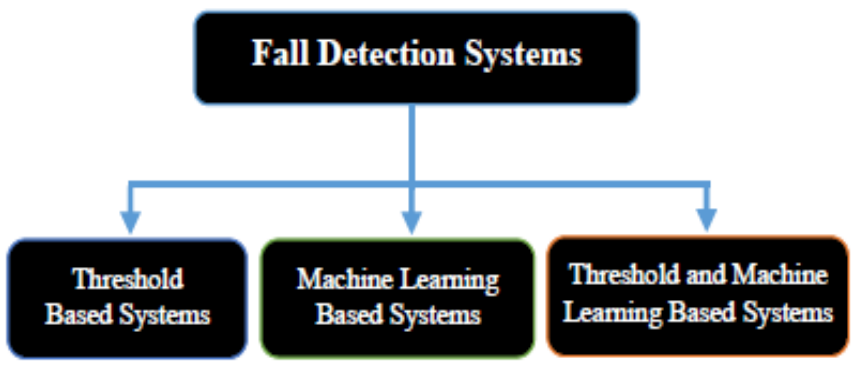

Figure 2. Fall detection systems classification

\subsection{Threshold based (TBA) fall detection systems}

Threshold based algorithms use a predefined fixed value to decide on a specific event. Threshold based algorithms require less computational power. They are also less complex than other sophisticated algorithms. The accuracy of the system largely depends on predefined threshold values.

The general block diagram of a threshold based fall detection system is illustrated in Figure 3. Human motion data is collected using the various smartphone sensors (i.e. Accelerometer, Gyroscope, Magnetometer, etc.). The collected sensor data is then compared with some preset values. These preset values can be specific sensor values, or differences between sensor readings, etc. A decision is taken based on whether the sensor values meet the required criteria defined in the system or not. If a fall detected, notification services are generally used to inform emergency services and mediators.

Victor et al. [26] proposed a fall detection system where the users were required to place a smartphone in their thigh pocket. The signal produced by the tri-axial accelerometer sensor was stored and converted into signal vector magnitudes to detect falls. The data along the Z-axis was analyzed to identify body inclination. The occurrence frequencies at the peak of the area of use were utilized as input parameters. High-Level Fuzzy Petri Net (HLFPN) was used for discriminating among walking, exercising and falling. After detecting a fall event, an alarm is raised, warning nearby individuals. This ensures immediate medical assistance. The reviewed model was successfully implemented in a smartphone featuring Android 2.3. Fall events were detected with $80 \%$ accuracy, which is comparatively low. The rate of misidentification of daily routines was $5.7 \%$. The model only worked when the device was put in the user's thigh pocket. Luis et al. [27] presented a fall detection system that used built-in sensors (accelerometer 
and gyroscope) of a smartphone. They proposed and integrated two separate algorithms. One of these was used to spot a fall event. The other algorithm can not only detect the location of the phone relative to the user's body (chests pocket, pants pockets, smartphone holster, etc.), but also detect if the user is talking, texting, and walking with the smartphone in hand. Thus, the working procedures and the threshold values change dynamically according to locations (total 6 locations) to produce better results. The total performance accuracy of the system was $81.3 \%$ in the case of fall detection and $72 \%$ in the case of the location selection algorithm. The best fall detection rate was achieved when the user was texting (95.8\%). The detection rate was $87.5 \%$ and $83.3 \%$ when the device was in thigh pocket, and chest pocket, respectively. The reviewed system shows low accuracy in the case of holster location because accelerometer reading does not shift significantly compared to other locations.

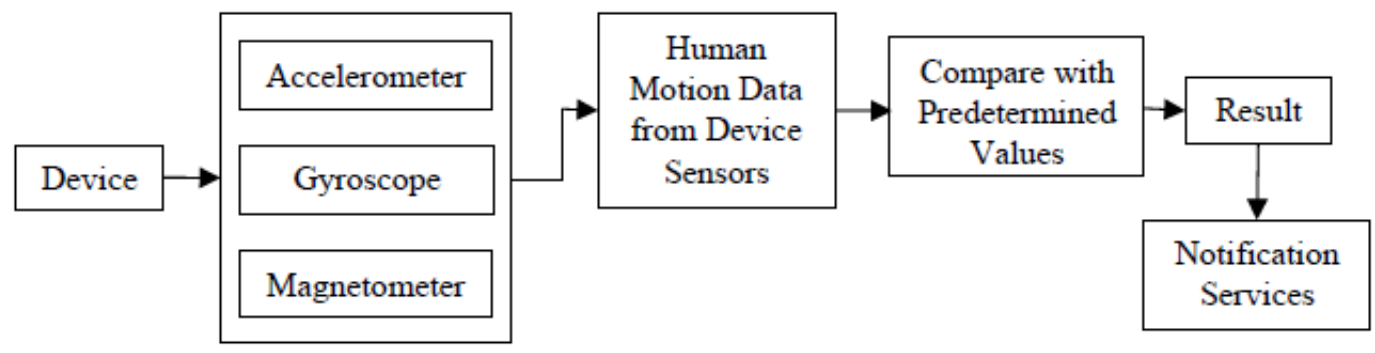

Figure 3. General block diagram of threshold based fall detection system

Ekachai et al. [28] presented a relatively simple but effective fall detection system. Their algorithm performed fewer calculations and thus could promptly differentiate between a fall and a normal activity of daily living (ADL). It would learn ADLs of a particular user and characterize the proper limit for the threshold value dynamically, depending on the user without using machine learning. The system would collect data using the built-in accelerometer of the smartphone. A fall is recognized by the algorithm if any $\mathrm{x}, \mathrm{y}$, or $\mathrm{z}$-axial acceleration value shifts approximately $10 \mathrm{~g}$ from the recorded ADL readings within a short period. A significant drawback of the system was that it only operated correctly when the phone was kept into the chest pocket or hung as a necklace ensuring that the front face of the phone was turned towards the body of the user. The accuracy and sensitivity of the system were not specified. Arkham et al. [29] proposed a prototype system for fall detection that uses smartphone technology. The system was relatively simple but produced better accuracy compared to others. Accelerometer coupled with a gyroscope was used as the main sensing device. The system could also differentiate between falls and activity of daily living (ADL) successfully. At first, the system determined if there was any instability of the user compared to some predefined values. Then, the system determined the angle (in degrees) to predict the posture of the user. Then the threshold value was set. The accuracy of the system was $93.3 \%$ and the error rate of fall detection was $2 \%$. Like the other reviewed systems, this system only worked if the smartphone was put in the waist position or waist pocket. Jin-Shyan et al. [30] introduced an upgraded threshold based fall detection mechanism. It was capable of differentiating falls from ADLs. It was also capable of identifying four directions of fall (front lateral, back lateral, left lateral, and right lateral). The device should be kept in the front pants pocket for collecting data by using the built-in accelerometer. Three predefined threshold values were set. Threshold-1 was used to detect actions that require very less or no movement like sitting down, squatting, standing. Threshold-2 was used to detect actions related to motion like running, movement along stairs and falling. Finally, the system was enhanced with more ADLs like jumping, lying down, etc. with the help of threshold-3. The system got an excellent accuracy and detection rate of about $99.38 \%$ and $96 \%$ which was very good for a TBA based system.

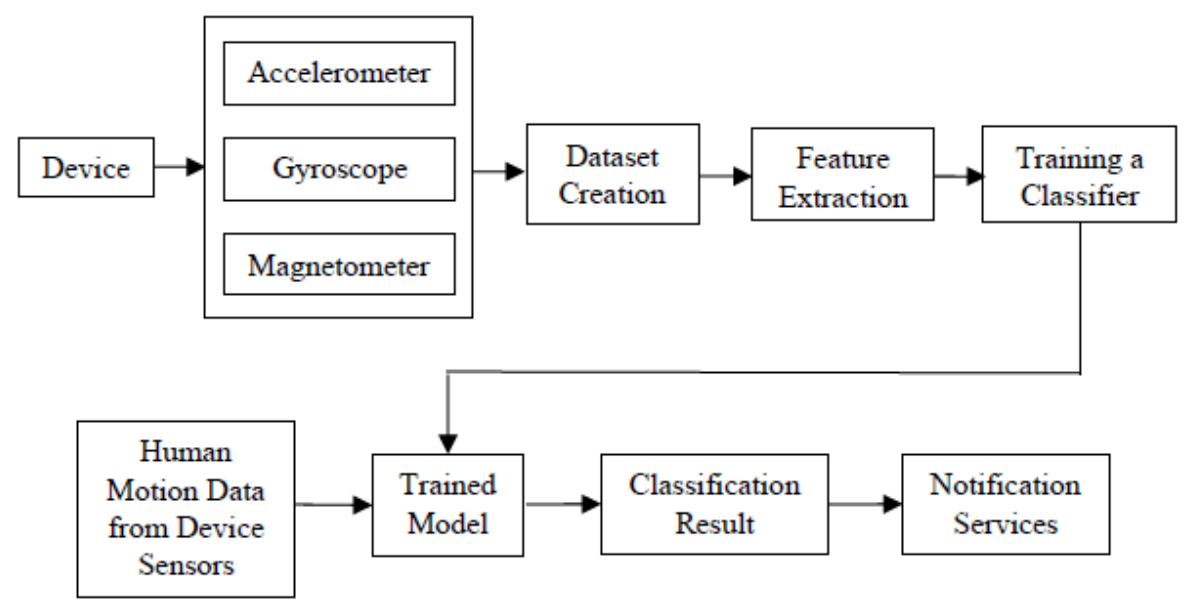

Figure 4. General block diagram of machine learning based fall detection system 
Hsieh et al. [31] presented a system that detected fall using a device similar to wristband along with a smartphone. Users could keep the phone in any pocket (Shirt or pant), even in purse or briefcase. The accelerometer of the wristband-type device would collect acceleration data and send it to the smartphone to process and decide if there was a fall. If data sent by the device was enough but not sufficient to ensure a fall then the smartphone acceleration data would be used to make the final decision. However, the accuracy of the wristwatch was better than the smartphone. But, the smartphone provided more accurate results in detecting ADLs. The accuracy was maximized and the error detection rate was minimized by combining the smartphone and wristwatch based systems.

\subsection{Machine learning (ML) based fall detection systems}

Machine learning (ML) is a sub-field of Artificial Intelligence (AI) where a computer can learn from sequences without the help of raw programming [32]. ML based technologies produce better results compared to threshold based algorithms.

The general block diagram of a ML based fall detection system is illustrated in Figure 4. Normally, the development of machine learning based fall detection systems can be divided into two parts: training and deployment. Generally, human motion data from smartphone sensors are collected and stored. Feature extraction methods are performed on the stored dataset for extracting meaningful features from the data. These features are then used to train a machine learning classifier model. Hyperparameter tuning is performed on the model parameters to make the classifier model more robust and to avoid common pitfalls such as overfitting and underfitting. The trained model can be then converted for direct use in smartphone application. The model can also be stored in a server for performing classification tasks using specific APIs [25]. If the pre-trained model is deployed in the smartphone, then human motion data from the sensors are classified locally. If the model is deployed in a server, then sensor data is sent to the server for classification. The model classifies the data and returns the results to the device [25]. The device then takes necessary measures based on the results.

Shahzad et al. [33] proposed a fall detection system for the elderly named "FallDroid" that detected the fall events using machine learning techniques. The proposed system used data that were collected through smartphone sensors. The smartphone could be put in both the waist and thigh position. The proposed system used novel techniques to successfully differentiate actions that are similar to fall (lying on a bed or sudden stop after running) and reduce false positives. The accuracy and sensitivity of the developed system were $97.8 \%$ and $99.5 \%$ for waist position, $97.8 \%$ and $99.5 \%$ for thigh positions. The system achieved a very low false alarm rate. 1 false alarm was detected per $59 \mathrm{~h}$ of usage. However, the system worked in a home environment and simple machine learning techniques were used whereas feature engineering could provide better results. John et al. [34] introduced a twostep fall detection system. The system used five smartphone sensors to read data, such as accelerometer, gyroscope, magnetometer, gravity, and, linear acceleration. Firstly, it performed multiclass classification, unlike other fall detection systems that used binary classification to identify the correct type of fall from the false one. They verified the system with five ML classifier algorithms: Support Vector Machine (SVM), K-Nearest Neighbor, Decision Tree, Random Forest, and, Naive Bayes. Among the tested algorithms, SVM was the most accurate. Secondly, the method produced a binary decision based on the multiclass prediction. The maximum accuracy of the system was about $95.65 \%$ and the maximum area under the region of convergence curve was 0.93 with the gyroscope sensor and SVM classifier. However, using deep learning methods the system could be improved further.

Pranesh et al. [35] introduced a fall detection system that used machine learning algorithms. They used the MobiFall dataset to train and validate their model. Moreover, they implemented feature extraction methods. This helped largely to reduce dimensionality. MobiFall dataset contains ADLs (standing, walking, jogging, jumping, walking up and down the stairs, and sitting on a chair) and four specific types of falls. For sensing data of body movements, an accelerometer, gyroscope, and magnetometer of a smartphone were used. The smartphone should be kept in the thigh pocket to operate correctly. Supervised machine learning models namely, Support Vector Machine (SVM), Naive Bayes, Least Squares Method (LSM), k-Nearest Neighbor (k-NN), and Artificial Neural Networks (ANNs) were used by them. K-NN performed best with accuracy, sensitivity, and specificity of $87.5 \%, 90.70 \%, 83.78 \%$, respectively. Michael et al. [36] used audio features of a smartphone to develop an automated fall detection system. The sound events from smartphones were collected at a sampling rate of $44.1 \mathrm{kHz}$. Features namely, the spectrogram, Mel Frequency Cepstral Coefficients (MFCCs), Linear Predictive coding (LPC), and Matching Pursuit (MP) of various fall and no-fall sound incidents were selected from experimental data. Four machine learning classifier algorithms (k-NN, SVM, LSM, and ANN) were tested on the extracted audio features to differentiate between a fall and a no-fall. The phone should be kept in the vicinity of the user $(5 \mathrm{~m}$ distance range) to operate. Spectrogram features with ANN classifier gave the best results with high sensitivity, specificity, and accuracy; all greater than $98 \%$. Though the system had significant advantages over other wearable systems, blind source separation (BSS) techniques might help to further upgrade the system performance in noisy conditions.

\subsection{Combined TBA and ML based systems}

In this section, systems that used a combination of both the TBA and the ML are reviewed. Mostly, TBA techniques are used for feature extraction and the ML algorithms are used for the classification of events. The primary goal is to beat the TBAs and MLs in recognizing falls and ADLs. These types of techniques require more processing, capacity and battery power compared to other techniques.

The general architecture of a combined fall detection system is presented in Figure 5. Combined fall detection systems use both preset sensor data and machine learning models for performing the classification task. Human motion data collected from smartphone sensors are stored and used to train a machine learning model. The trained model is then deployed in the device or a server. The combined system architecture can be arranged in many ways. The threshold based algorithm can be used for only detecting possible fall events. 


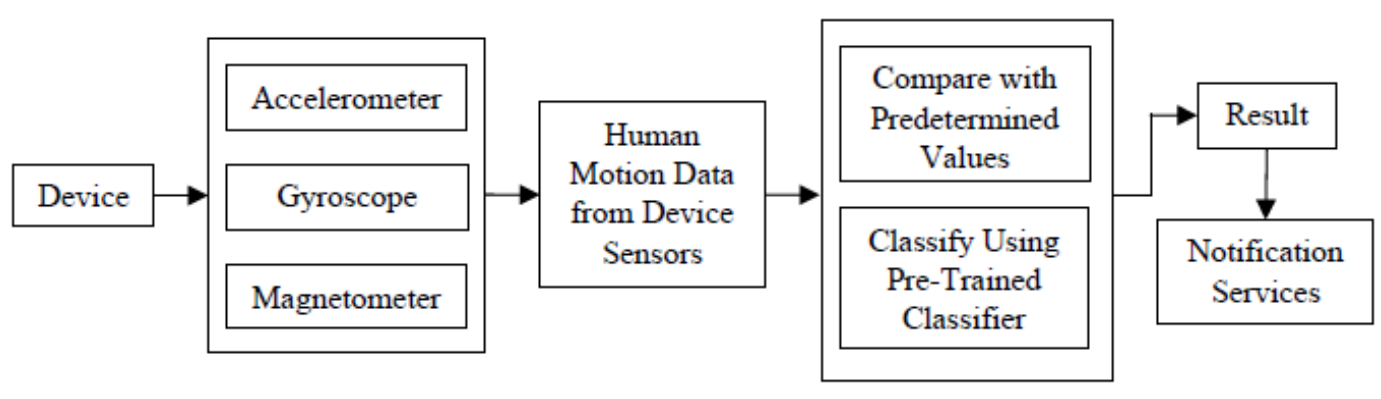

Figure 5. General block diagram of combined fall detection system

Table 1. Summary of the reviewed fall detection systems

\begin{tabular}{|c|c|c|c|c|c|c|c|c|c|c|c|c|c|c|c|c|c|c|c|}
\hline 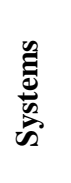 & 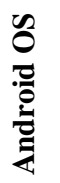 & $\stackrel{2}{2}$ & 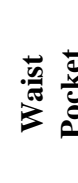 & 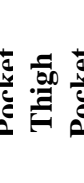 & 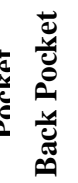 & $\stackrel{\mathscr{D}}{\leftrightarrows}$ & 武 & 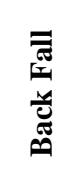 & 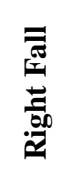 & لَّ & 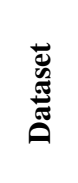 & 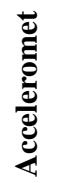 & 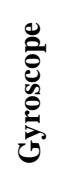 & & 氡 & $\stackrel{\nexists}{\nexists}$ & 氖 & 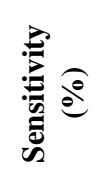 & $\widehat{\overparen{E}}$ \\
\hline [26] & $\checkmark$ & $\checkmark$ & $\checkmark$ & $\checkmark$ & $\checkmark$ & $x$ & $\checkmark$ & $\checkmark$ & $\checkmark$ & $\checkmark$ & N/A & $\checkmark$ & $x$ & $x$ & N/A & 2013 & N/A & N/A & 80 \\
\hline [27] & $\checkmark$ & $x$ & $\checkmark$ & $\checkmark$ & $\checkmark$ & $x$ & $x$ & $x$ & $x$ & $x$ & $\checkmark$ & $\checkmark$ & $x$ & $x$ & TBA & 2014 & $\begin{array}{l}67- \\
100\end{array}$ & N/A & $\begin{array}{c}83.3- \\
95.8\end{array}$ \\
\hline [28] & $\checkmark$ & $\times$ & $x$ & $x$ & $x$ & $x$ & $\checkmark$ & $\checkmark$ & $\checkmark$ & $\checkmark$ & $\checkmark$ & $\checkmark$ & $x$ & $x$ & TBA & 2014 & N/A & N/A & N/A \\
\hline [29] & $\checkmark$ & $x$ & $\times$ & $x$ & $\times$ & $x$ & $\checkmark$ & $\checkmark$ & $\checkmark$ & $\checkmark$ & $\checkmark$ & $\checkmark$ & $\checkmark$ & $x$ & TBA & 2014 & N/A & N/A & $\begin{array}{c}86.67- \\
100\end{array}$ \\
\hline [30] & $\checkmark$ & $x$ & $\checkmark$ & $\checkmark$ & $\checkmark$ & $\checkmark$ & $\checkmark$ & $\checkmark$ & $\checkmark$ & $\checkmark$ & $\checkmark$ & $\checkmark$ & $\checkmark$ & $\checkmark$ & TBA & 2017 & $\begin{array}{l}87- \\
100\end{array}$ & $73-100$ & N/A \\
\hline [31] & $\checkmark$ & $x$ & $\checkmark$ & $\checkmark$ & $\checkmark$ & $x$ & $\checkmark$ & $\checkmark$ & $\checkmark$ & $\checkmark$ & $\checkmark$ & $\checkmark$ & $x$ & $\checkmark$ & TBA & 2019 & N/A & N/A & 99.38 \\
\hline [33] & $\checkmark$ & $x$ & $\checkmark$ & $\checkmark$ & $\checkmark$ & $x$ & $\checkmark$ & $\checkmark$ & $\checkmark$ & $\checkmark$ & $\checkmark$ & $\checkmark$ & $\checkmark$ & $\checkmark$ & $\begin{array}{c}\text { TBA, MKL-SVM, SVM, } \\
\text { ANN, K-NN, Naive } \\
\text { Bayes }\end{array}$ & 2018 & $\begin{array}{c}88- \\
95.2\end{array}$ & $\begin{array}{l}95.8- \\
99.5\end{array}$ & $\begin{array}{l}91.7- \\
97.8\end{array}$ \\
\hline [34] & $\checkmark$ & $x$ & N/A & N/A & N/A & N/A & N/A & N/A & N/A & N/A & $\checkmark$ & $\checkmark$ & $\checkmark$ & $\checkmark$ & $\begin{array}{l}\text { TBA, SVM, DT, RF, } \\
\text { KNN, Naive Bayes }\end{array}$ & 2019 & N/A & N/A & $\begin{array}{l}78.63- \\
96.65\end{array}$ \\
\hline [35] & $\checkmark$ & $x$ & $\checkmark$ & $\checkmark$ & $\checkmark$ & $x$ & $\checkmark$ & $\checkmark$ & $\checkmark$ & $\checkmark$ & $\checkmark$ & $\checkmark$ & $\checkmark$ & $\checkmark$ & $\begin{array}{c}\text { Naive Bayes, SVM, A- } \\
\text { NN, LSM }\end{array}$ & 2016 & 83.78 & 90.70 & 87.5 \\
\hline [36] & $\checkmark$ & $x$ & N/A & N/A & N/A & N/A & N/A & N/A & N/A & N/A & $\checkmark$ & $\checkmark$ & $\checkmark$ & $x$ & $\begin{array}{l}\text { K-NN, LSM, SVM, } \\
\text { ANN }\end{array}$ & 2015 & 98 & 98 & 98 \\
\hline [37] & $\checkmark$ & $x$ & $\checkmark$ & $\checkmark$ & $x$ & $x$ & N/A & N/A & N/A & N/A & & $\checkmark$ & $x$ & $x$ & $\begin{array}{l}\text { TBA, KNN, ANN, SVM, } \\
\text { J48 }\end{array}$ & 2017 & 97.07 & 95.52 & 91.83 \\
\hline [38] & $\checkmark$ & $x$ & N/A & $\checkmark$ & N/A & N/A & $x$ & $x$ & $x$ & $x$ & $\checkmark$ & $\checkmark$ & $x$ & $x$ & $\begin{array}{l}\text { TBA, ANN, Fuzzy } \\
\text { Logic, AdaBoost }\end{array}$ & 2015 & N/A & N/A & N/A \\
\hline [39] & $\checkmark$ & $x$ & $\checkmark$ & $\checkmark$ & $\checkmark$ & $x$ & $\checkmark$ & $\checkmark$ & $\checkmark$ & $\checkmark$ & $\checkmark$ & $\checkmark$ & $\checkmark$ & $x$ & $\begin{array}{c}\text { TBA, DT, K-NN, Naive } \\
\text { Bayes }\end{array}$ & 2014 & $\begin{array}{l}77.5- \\
96\end{array}$ & $\begin{array}{l}77.6- \\
97.3\end{array}$ & $\begin{array}{l}77.5- \\
93.7\end{array}$ \\
\hline
\end{tabular}

* N/A = Not Appropriately defined

The possible fall events motion data can be then sent to the pre-trained classifier model for final classification. This approach reduces computational and time complexity as the model does not classify every motion event. The model is only used for properly classifying possible fall motion events. Another approach is to use both the threshold based algorithm and the classifier for classifying each motion data. If both of them detect a fall event, the notification services are used to take the necessary measures.

Panagiotis et al. [37] proposed a TBA technique for fall detection whose performance was significantly upgraded by using a machine learning classifier algorithm k-Nearest Neighbor (k-NN). The system is made up of four parts. Firstly, the smartphone accelerometer collected data that were preprocessed using a series of magnitude and time thresholds to identify fall-like events. Secondly, Features were extracted from these events, which were used to train the k-NN classifier algorithm to differentiate between fall and ADLs. Thirdly, there was a module to control the activation level of the sensor as high battery drainage is a concern in ML based systems. And lastly, a component was added that adjusted the model with the user activity patterns. The accuracy of the system was 
moderately high. The sensitivity and specificity of the system were $97.53 \%$ and $94.89 \%$, respectively. However, the system was not tested in real-life environments, and battery usage is a huge concern. Stefan et al. [38] introduced a smartphone based fall detection system that characterized both TBA and ML. Three sensors were used for sensing: angular rate of change using a gyroscope, maximum acceleration using an accelerometer, maximum attitude change using magnetometer. These values were then compared with the following preset thresholds: angular rate of change ( $\mathrm{THl}$ ), maximum acceleration (TH2), and maximum attitude change (TH3) for sensing a fall. Five basic ADLs: 1) comfortable walking, 2) stand-to-seated posture, 3) seated-to-standing posture, 4) pivoting at the waist to pick up an object, and 5) stand-toseated-to-laying transition didn't violate any thresholds thus ensured no false positives. The system implemented ML algorithms, namely artificial neural networks, fuzzy logic, and adaptive meta-algorithms (e.g., AdaBoost) to identify different events based on the tri-axial accelerometer. These designs were extended by the algorithmic execution of triaxial gyroscopes in real-time neural networks, joined with adaptive meta-algorithms. These improvements made the system robust. These also helped the system reducing the rate of false positives and false negatives.

An unobtrusive fall detection system was introduced by Bruno et al. [39] that was able to detect all the phases of fall events. It used a combined form of information generated from machine learning classification applied in a state machine algorithm. The smartphone should be kept in the user's belt or pocket and a built-in accelerometer would continuously take the data in a state machine that recognized the fall phases in successive order. Three classifier algorithms were tested: decision tree, K-NN, Naive Bayes. The decision tree algorithm achieved the best results. The thresholds and features of the acceleration values had been determined using decision trees, after a comparison of diverse machine learning classifiers. The system was able to produce a high accuracy rate of about $97.5 \%$ for the pocket and belt usage. However, considerable improvements could be achieved by adding a temporal reference, where different thresholds and analysis were employed according to the stages of the fall.

\section{DISCUSSION AND RECOMMENDATION FOR FUTURE WORK}

The summary of the review literature is based on the position of the device, algorithms used, accuracy, sensitivity, etc. The summary of the reviewed systems is depicted in Table 1. All the systems hold some basic criteria and give the desired result with certain accuracy. But some of the systems performed better than others in some specific situations. The threshold based system employing a wrist-bound accelerometer [31] and the machine learning based system employing five smartphone sensors [34] obtained accuracy scores of $99 \%$ and $96 \%$, respectively. The threshold based system based on accelerometer and gyroscope achieved the highest accuracy [29]. The machine learning based system employing audio features of smartphones [36] has accuracy, sensitivity, and specificity greater than $98 \%$. However, fall type specification or device position in the human body is not specified. The threshold based system using accelerometer readings [30] supports fall detection even if the smartphone is put in a purse, which is very exceptional from other systems.
However, no accuracy metrics are present. The combined machine learning and threshold based system employing accelerometer, gyroscope, and magnetometer didn't provide any accuracy measures [38]. Future systems can be made better by merging the strengths of the latest available systems and taking care of their weaknesses.

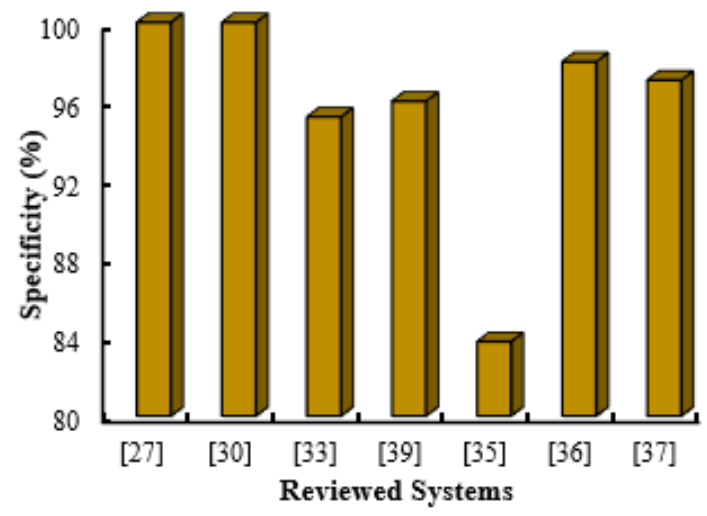

Figure 6. Specificity of the reviewed systems

Figure 6 shows a graphical representation of the specificity of the reviewed systems. Some systems didn't appropriately present the specificity of their system. On the other hand, some systems defined the specificity of their system for every position of the device. The threshold based system using accelerometer and gyroscope [27] and the threshold based system employing accelerometer [30] achieved $100 \%$ specificity for specific positions. However, the specificity for other positions was comparatively low. ML based systems showed better specificity than others. The machine learning based system employing accelerometer, gyroscope, and magnetometer sensors achieved the highest specificity of $98 \%$ [35].

Figure 7 shows a graphical comparison of the sensitivity of the systems. Some systems didn't appropriately present the sensitivity of their system. Most of the systems were able to acquire more than $90 \%$ sensitivity. The machine learning based system employing smartphone sensors [33] achieved 99.5\% specificity for a specific position and more than $95.8 \%$ specificity for other positions.

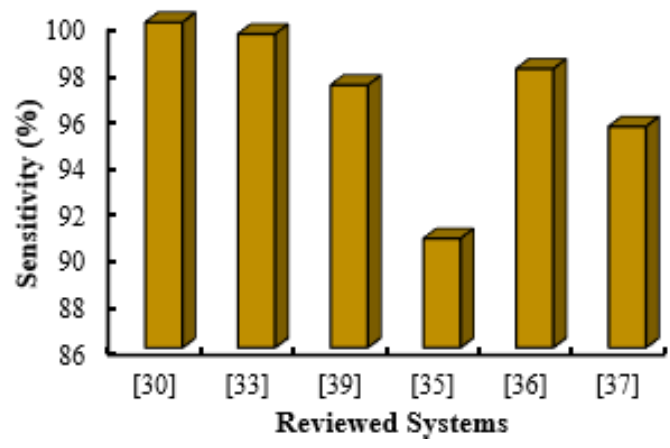

Figure 7. Sensitivity of the reviewed systems

Figure 8 shows the pictorial view of the accuracy of the reviewed systems. The accuracy of the systems fluctuated a lot based on the technology used in the systems as well as the position of the device. For TBA based systems, whereas the system employing tri-axial accelerometers [26] achieved $80 \%$ accuracy score, the wrist-bound accelerometer based system [31] achieved $99.38 \%$ accuracy score. ML based systems 
performed better for specific positions but not for every position.

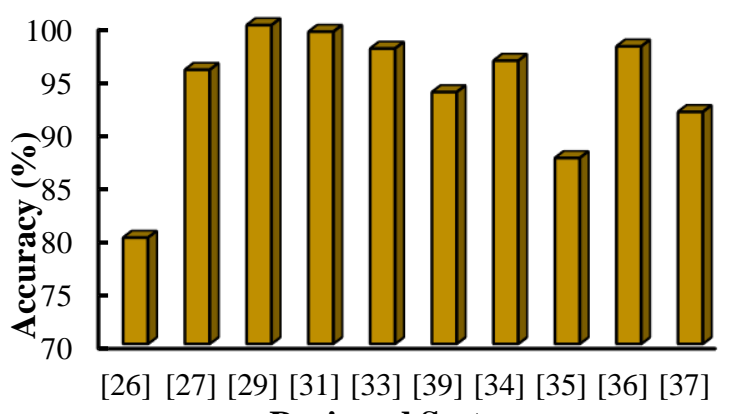

Reviewed Systems

Figure 8. Accuracy of the reviewed systems

As smartphones usually have a limited battery and resource power, an optimized system is desired. During charging, the user is prone to experience a fall that can't be detected. Most of the systems are developed considering only Android OS based smartphones. Further systems should be developed for other widely used mobile operating systems such as iOS, Windows OS, etc. Threshold based algorithms are easier to develop for multiple operating systems. Machine learning based algorithms are not widely supported in all mobile operating systems yet. Thus, threshold based algorithms are easier to port to other operating systems.

Adaptive TBA should be used for developing personspecific systems. Adaptive threshold based systems can dynamically adjust themselves for specific users. These methods should be enhanced by using ML algorithms. While using ML algorithms, data overfitting must be eliminated. A new ML algorithm named augmented random search, which is used for shallow learning, can be widely used for better results and learning optimization. The combination of TBA and ML systems have achieved the best results. Speed of walking, health info, and physical fitness can be determined by combining these two algorithms.

\section{CONCLUSION}

This paper represented a summary of smartphone based fall detection systems based on either one of TBA, ML techniques or a combination of both. The systems employing threshold based algorithms had a low percentage of accuracy but they require less computational power and produce fast output. Most of the systems employing machine learning algorithms required the smartphone to be kept in a specific position relative to the body to operate correctly. Machine learning based systems produced better accuracy both in detecting fall and ADLs but was less reliable for real-time devices. Machine learning based systems also drain the batteries faster. The Combined technology of threshold based and machine learning based systems produced good accuracy but with the cost of high computational power usage.

\section{REFERENCES}

[1] Cambridge Online English Dictionary, https://dictionary.cambridge.org/dictionary/english/fall. accessed on: June. 1, 2019.
[2] Bergen, G. (2016). Falls and fall injuries among adults aged $\geq 65$ years-United States, 2014. Morbidity and Mortality Weekly Report (MMWR), 65(37): 993-998. https://dx.doi.org/10.15585/mmwr.mm6537a2

[3] Ambrose, A.F., Cruz, L., Paul, G. 2015. Falls and fractures: A systematic approach to screening and prevention. $\quad$ Maturitas, 82(1): 85-93. https://doi.org/10.1016/j.maturitas.2015.06.035

[4] Ambrose, A.F., Paul, G., Hausdorff, J.M. (2013). Risk factors for falls among older adults: A review of the literature. $\quad$ Maturitas, 75(1): 51-61. https://doi.org/10.1016/j.maturitas.2013.02.009

[5] Stevens, J.A., Corso, P.S., Finkelstein, E.A., Miller, T.R. (2006). The costs of fatal and non-fatal falls among older adults. Injury Prevention, 12(5): 290-295. https://doi.org/10.1136/ip.2005.011015

[6] Talbot, L.A., Musiol, R.J., Witham, E.K., Metter, E.J. (2005). Falls in young, middle-aged and older community dwelling adults: perceived cause, environmental factors and injury. BMC Public Health, 5(1): 86. https://doi.org/10.1186/1471-2458-5-86

[7] Nihseniorhealth: Causes and Risk Factors [Online]. http://nihseniorhealth.gov/falls/causesandriskfactors/01. html/, accessed on: June. 1, 2019.

[8] Lord, S.R., Menz, H.B., Sherrington, C. (2006). Home environment risk factors for falls in older people and the efficacy of home modifications. Age Ageing, 35(Suppl 2): ii55-ii59. https://doi.org/10.1093/ageing/afl088

[9] Homann, B., Plaschg, A., Grundner, M., Haubenhofer, A., Griedl, T., Ivanic, G., Hofer, E., Fazekas, F., Homann, C.N. (2013). The impact of neurological disorders on the risk for falls in the community dwelling elderly: A casecontrolled study. BMJ Open, 3(11): e003367. https://dx.doi.org/10.1136/bmjopen-2013-003367

[10] World Health Organization and Alzheimer's Disease International. Dementia: A Public Health Priority. World Health Organization; Geneva, Switzerland: 2012, accessed on: June. 1, 2019.

[11] Centers for Disease Control and Prevention, National Center for Injury Prevention and Control.[online]. https://www.cdc.gov/injury/wisqars/, accessed on: Jul. 1, 2019.

[12] "Falls," World Health Organization. [Online]. http://www.who.int/mediacentre/factsheets/fs344/en/, accessed on: Jul. 1, 2019.

[13] Tacconi, C., Mellone, S., Chiari, L. (2011). Smartphonebased applications for investigating falls and mobility. Proceedings of the 5th International Conference on Pervasive Computing Technologies for Healthcare (Pervasive Health); Dublin, Ireland, pp. 258-261. https://dx.doi.org/10.4108/icst.pervasivehealth.2011.246 060

[14] Igual, R., Medrano, C., Plaza, I. (2013). Challenges, issues and trends in fall detection systems. Bio Medical Engineering OnLine, 12(1): 1-24. https://doi.org/10.1186/1475-925X-12-66

[15] "Mobile phone users worldwide 2015-2020," Statista. [Online]. https://www.statista.com/statistics/274774/forecast-ofmobile-phone-users-worldwide/, accessed on: Oct. 27, 2019.

[16] "Smartphone users worldwide 2016-2021," Statistia. [Online]. https://www.statista.com/statistics/330695/number-of- 
smartphone-users-worldwide/, accessed on Oct. 27, 2019.

[17] Khan, S.S., Hoey, J. (2017). Review of fall detection techniques: A data availability perspective. Medical Engineering \& Physics, 39: 12-22. https://doi.org/10.1016/j.medengphy.2016.10.014

[18] Mellone, S., Tacconi, C., Chiari, L. (2012). Validity of a smartphone-based instrumented timed up and go. Gait \& Posture, 36(1): 163-165 https://doi.org/10.1016/j.gaitpost.2012.02.006

[19] Albert, M.V., Kording, K., Herrmann, M., Jayaraman, A. (2012). Fall classification by machine learning using smobile phones. Plos One, 5: e36556. https://doi.org/10.1371/journal.pone.0036556

[20] Vogt, P., Kuhn, J. (012). Analyzing free fall with a smartphone acceleration sensor. Phys. Teach. 182(2012): 182-183. https://doi.org/10.1119/1.3685123

[21] Casilari, E., Luque, R., Morón, M.J. (2015). Analysis of android device based solutions for fall detection. Sensors, 15(8):

$17827-17894$ https://doi.org/10.3390/s150817827

[22] Figueiredo, I.N., Leal, C., Pinto, L., Bolito, J., Lemos, A. (2016). Exploring smartphone sensors for fall detection. The Journal of Mobile User Experience, (2). https://doi.org/10.1186/s13678-016-0004-1

[23] Anguita, D., Ghio, A., Oneto, L., Parra, X., Reyes-Ortiz, J.L. (2013). Energy efficient smartphone-based activity recognition using fixed-point arithmetic. Journal of Universal Computer Science, 19(9): 1295-1314. https://doi.org/10.3217/jucs-019-09-1295

[24] Igual, R., Medrano, C., Plaza, I. (2013). Challenges, issues and trends in fall detection systems. Biomedical Engineering Online, 12(1): 66 https://doi.org/10.1186/1475-925X-12-66

[25] Nooruddin, S., Islam, M.M., Sharna, F.A. (2019). An IoT based device-type invariant fall detection system. Internet of Things, 9: 100130. https://doi.org/10.1016/j.iot.2019.100130

[26] Shen, V.R.L., Lai, H.Y., Lai, A.F. (2013). Application of High-Level Fuzzy Petri Nets to fall detection system using smartphone. 2013 International Conference on Machine Learning and Cybernetics, Tianjin, pp. 1429. 1435. https://doi.org/10.1109/ICMLC.2013.6890807

[27] Colón, L.N.V., DeLaHoz, Y., Labrador, M. (2014). Human fall detection with smartphones. 2014 IEEE Latin-America Conference on Communications (LATINCOM), Cartagena de Indias, pp. 1-7. https://doi.org/10.1109/LATINCOM.2014.7041879

[28] Thammasat, E., Chaicharn, J. (2012). A simply falldetection algorithm using accelerometers on a smartphone. The 5th 2012 Biomedical Engineering International Conference, Ubon Ratchathani, pp. 1-4. https://doi.org/10.1109/BMEiCon.2012.6465471

[29] Rakhman, A.Z., Nugroho, L.E., Widyawan,
Kurnianingsih. (2014). Fall detection system using accelerometer and gyroscope based on smartphone. 2014 The 1st International Conference on Information Technology, Computer, and Electrical Engineering, Semarang, pp. 99-104. https://doi.org/10.1109/ICITACEE.2014.7065722

[30] Lee, J., Tseng, H. (2019). Development of an enhanced threshold-based fall detection system using smartphones with built-in accelerometers. In IEEE Sensors Journal, 19(18): 8293-8302. https://doi.org/10.1109/JSEN.2019.2918690

[31] Hsieh, S., Yang, C., Li, H. (2017). Combining wristbandtype devices and smartphones to detect falls. 2017 IEEE International Conference on Systems, Man, and Cybernetics (SMC), Banff, AB, pp. 2373-2377. https://doi.org/10.1109/SMC.2017.8122977

[32] Forth, K., Aiden, E.L. (2019). Identifying fall risk using machine learning algorithms. U.S. Patent.

[33] Shahzad, A., Kim, K. (2019). FallDroid: An automated smart-phone-based fall detection system using multiple kernel learning. In IEEE Transactions on Industrial Informatics, 15(1): 35-44. https://doi.org/10.1109/TII.2018.2839749

[34] Dogan, J.C., Hossain, M.S. (2019). A novel two-step fall detection method using smartphone sensors. 2019 IEEE International Conference on Smart Computing (SMARTCOMP), Washington, DC, USA, pp. 434-438. https://doi.org/10.1109/SMARTCOMP.2019.00083

[35] Vallabh, P., Malekian, R., Ye, N., Bogatinoska, D.C. (2016). Fall detection using machine learning algorithms. 2016 24th International Conference on Software, Telecommunications and Computer Networks (SoftCOM), Split, $\quad$ pp. https://doi.org/10.1109/SOFTCOM.2016.7772142

[36] Cheffena, M. (2016). Fall detection using smartphone audio features. In IEEE Journal of Biomedical and Health Informatics, 20(4): 1073-1080. https://doi.org/10.1109/JBHI.2015.2425932

[37] Tsinganos, P., Skodras, A. (2017). A smartphone-based fall detection system for the elderly. Proceedings of the 10th International Symposium on Image and Signal Processing and Analysis, Ljubljana, pp. 53-58. https://doi.org/10.1109/ISPA.2017.8073568

[38] Madansingh, S., Thrasher, T.A., Layne, C.S., Lee, B. (2015). Smartphone based fall detection system. 2015 15th International Conference on Control, Automation and Systems (ICCAS), Busan, pp. 370-374. https://doi.org/10.1109/ICCAS.2015.7364941

[39] Aguiar, B., Rocha, T., Silva, J., Sousa, I. (2014). Accelerometer-based fall detection for smartphones. 2014 IEEE International Symposium on Medical Measurements and Applications (MeMeA), Lisboa, pp. 1-6. https://doi.org/10.1109/MeMeA.2014.6860110 